\title{
The Promise of Liraglutide in Type 2 Diabetes Mellitus
}

\section{Tarek Elshourbagy*}

Faculty of Medicine-Cairo University, Egypt

*Corresponding Author: Tarek Elshourbagy, Faculty of Medicine-Cairo University, Egypt.

Received: September 13, 2019; Published: September 20, 2019

DOI: 10.31080/ASPS.2019.03.0401

\begin{abstract}
Diabetes mellitus (DM) is a chronic medical condition which is charachterized by hyperglycemia. It has very serious acute complications such as Diabetic Ketoacidosis (DKA) and Hyperosmotic Hyperglycemic Non ketotic Syndrome (HHNS). Also it has serious long term complications such as macroangiopathies, microangiopathies, neuropathies and others. Many drugs have been developed to combat this disease. However, it remains controlled in fewer than $50 \%$ of patients in the U.S [1]. The Incretin mimetics as Liraglutide are new agents which show great results in DM type 2. In this article we will discuss the mechanisms of action, types, outcome and adverse effects of Liraglutide.
\end{abstract}

Keywords: Liraglutide; Diabetes Mellitus; Type 2

\section{Introduction}

Diabetes mellitus (DM) is one of the most common and serious metabolic disorders and probably one of the oldest diseases known to human;it was first described in Egyptian manuscript about 3000 years ago [2]. There are two types of DM; type 1 in which the pancreas fails to produce Insulin and type 2 in which there is Insulin resistance and decreased Insulin sensitivity. It is estimated that 366 million people had DM in 2011. By 2030 this would have risen to 552 millions. DM caused 4.6 million death in 2011 [3].

Although many drugs are available for managing DM type 2, it remains controlled in fewer than $50 \%$ of people in the U.S [1], hence more efforts are needed to achieve the recommended ADA and AAACE HbA1c goals below 7\% and 6.5\% respectively.

The Incretin mimetics is a class of medications which was introduced to the market in 2005 as Exenatide [4]. Liraglutide was approved by the FDA in January 2010 as an adjuvant to diet and exercise in adults with type 2 DM [5].

\section{Mechanisms of action}

Liraglutide is a GLP-1 analogue with $97 \%$ amino acids sequence homology to GLP-1. It works on GLP-1 receptors in Beta and Alpha cells in pancreatic islets. These receptors are coupled to the enzyme Adenylate Cyclase; this causes increase in cAMP, activation of Protein Kinase A and increase in Calcium level [6] to stimulate the glucose dependent release of Insulin and inhibit the glucose dependet release of Glucagon [7]. Many studies showed that Liraglutide can control glucose level via slowing gastric empyting [8] and increase satiety through activation of different areas in the hind brain and probably via preserving free Leptin level [9]. This could be attributed to the weight loss which occurs with usage of Liraglutide, hence recent studies recommend using it for Obesity $[8,9]$.

Experiments were carried out on animal models and revealed that Liraglutide stimulates beta cell prolifeation and pancreatic islet neogenesis.It also protects pancreatic beta cell line(BTC-6 cells) against apoptosis [10]. 


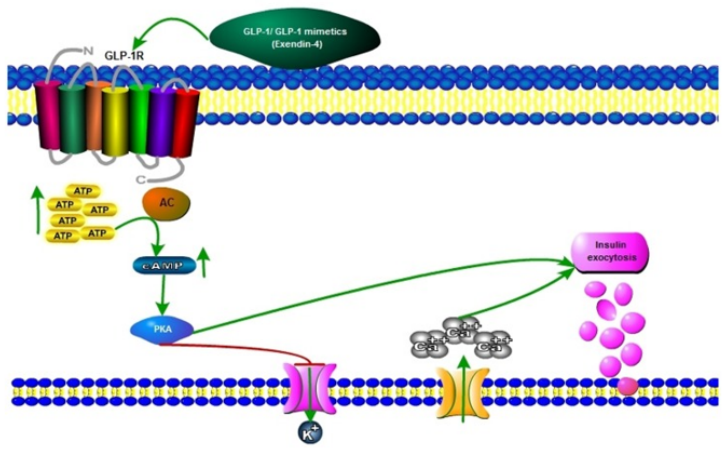

Figure 1

Types of liraglutide

Low dose: VICTOZA 1.2mg Injection

High dose: SAXENDA 3MG 15

Clinical effects of liraglutide

Improvement of glycemic control

Liraglutide has been tried out as monotherapy and HbA1c reduced from baseline by $0.84 \%$ with daily doses of $1.2 \mathrm{mg}$ and by $1.14 \%$ with daily dose of $1.8 \mathrm{mg}$ [11].

A clinical trial was carried out in diabetic patients with high CVS risk taking Liraglutide and it showed reduction of $\mathrm{HbA} 1 \mathrm{c}$ by $0.40 \%$ after 36 months of treatment ( $\mathrm{CI}=0.45 \%-0.34 \%)$ with decreased risk of hypoglycemia in Comparison with patients who received Placebo [12].

Another clinical trial was carried out on patients with $\mathrm{HbA1c}$ ranged at baseline from $8.4 \%$ to $8.5 \%$ and it showed decrease up to $1.1 \%$ with using Liraglutide and Glimepride [13].

Ar clinical study was carried out on Liraglutide as a 2nd line therapy with Metformin and it showed decrease in HbA1c values $0.7 \%$ with low dose $(0.6 \mathrm{mg} /$ day) and $1 \%$ for high dose $(1.2$ and $1.8 \mathrm{mg} /$ day) [14].

A clinical study has been carried out on LIraglutide as a 3rd line therapy with Metformin and Rosiglitazone and it showed reduction in $\mathrm{HbA} 1 \mathrm{c}$ by $1.5 \%$ for both daily doses of 1.2 and $1.8 \mathrm{mg} /$ day [15].

\section{Weight loss}

Recently, Liraglutide has been used for weight loss and it showed promising results.
Five randomized, placebo-controlled trials of liraglutide for weight management were identified. In addition to recommended diet and physical activity, liraglutide consistently resulted in a 4 to $6 \mathrm{~kg}$ weight loss [16].

\section{Cardiovasuar and lipid profile}

Use of liraglutide was associated with lowering the risk of MACE (major associated cardiovascular events) and death in patients with type2 DM \&high cardiovascular risk using basal insulin [17].

It lowers systolic blood pressure and lipid profile [18].

\section{Adverse effects}

Unfortunately like many drug agents, Liraglutide has been linked to some adverse effects; The most common (prevelance greater than 5\%) are nausea, hypoglycemia, dirrhea, constipation, headache, dyspepsia, fatigue, abdominal pain and increased level of Lipase enzyme [19]. A randomized study has been carried out on a population with type 2 DM at high CVS risk and it showed that acute pancreatitis occurred in $0.4 \%$ in patients treated with Liraglutide [20]. This can been assessed by increase in pancreatic enzymes level and Ultrasound in which the pancreas appears as a hypoechoic mass (Figure 2).

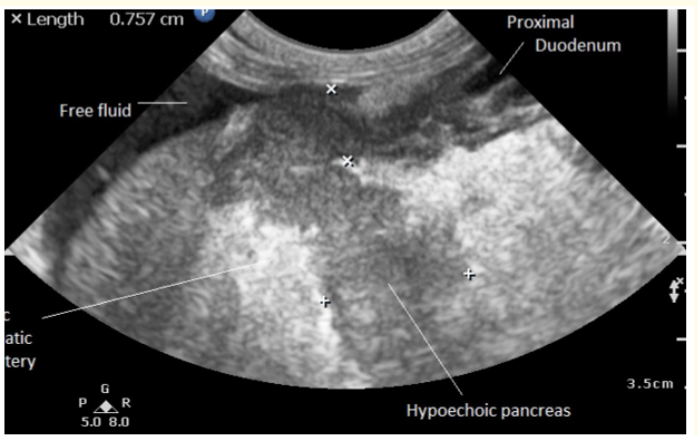

Figure 2

It is marked with a black box warning about the risk of medullary Thyroid Carcinoma as it has been shown to increase Thyroid C-cell tumors in rats and mice. However, a study has been carried out over 6000 patients and it showed no increased risk for MTC in humans [20]. 


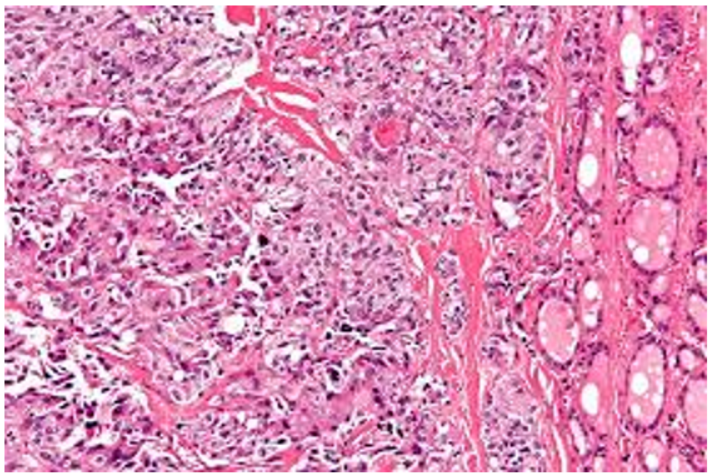

Figure 3

\section{Conclusion}

The therapeutic promise of Liraglutide is now evident from clinical data.It showed the potential to achieve good glycemic control in type 2 DM, weight loss and improvement in Cardiovascular and lipid profile. However, challenges remain because of its high cost, the need for injection and other adverse effects.

\section{Bibliography}

1. Resnick HE., et al. "Achievement of American Diabetes Association clinical practice recommendations among U.S. adults with diabetes, 1999-2002: The National Health and Nutrition Examination Survey". Diabetes Care 29 (2006): 531-537.

2. Ahmed AM. "History of diabetes mellitus". Saudi Medical Journal 23 (2002): 373-378.

3. Global burden of diabetes. "International Diabetes federation". Diabetic atlas fifth edition 2011, Brussels

4. Amylin and Lilly announce FDA approval of Byetta (Exenatide) injection (2005).

5. Princeton NJ. Victoza (liraglutide), product information. Novo Nordisk Inc.

6. Martín G Vila Petroff., et al. "Glucagon-Like Peptide-1 Increases cAMP but Fails to Augment Contraction in Adult Rat Cardiac Myocytes" Circulation Research 9 (2001): 445-452.

7. Shinobu Matsumoto., et al. "Effects of liraglutide on postprandial insulin and glucagon responses in Japanese patients with type 2 diabetes" (2013).

8. J van Can., et al. "Effects of the once-daily GLP-1 analog liraglutide on gastric emptying, glycemic parameters, appetite and energy metabolism in obese, non-diabetic adults" (2014).
9. Iepsen EW., et al. "Liraglutide for Type 2 diabetes and obesity: a 2015 update" (2015).

10. Katerina Kapodistria., et al. "Liraglutide, a human glucagonlike peptide-1 analogue, stimulates AKT-dependent survival signalling and inhibits pancreatic $\beta$-cell apoptosis" (2018).

11. Garber A., et al. "Liraglutide versus glimepiride monotherapy for Type 2 diabetes (LEAD-3 Mono): a randomised, 52-week, Phase III, double-blind, parallel-treatment trial”. Lancet 373 (2009): 473-481.

12. Bernard Zinman., et al. "Liraglutide and Glycaemic Outcomes in the LEADER Trial” (2018).

13. Marre M., et al. "Liraglutide, a once-daily human GLP-1 analogue, added to a sulphonylurea over 26 weeks produces greater improvements in glycaemic and weight control compared with adding rosiglitazone or placebo in subjects with Type 2 diabetes (LEAD-1 SU)". Diabetic Medicine 26.3 (2009): 268-278.

14. Nauck M., et al. "Efficacy and safety comparison of liraglutide, glimepiride, and placebo, all in combination with metformin, in Type 2 diabetes: the LEAD (liraglutide effect and action in diabetes)-2 study". Diabetes Care 32 (2009): 84-90.

15. Zinman B., et al. "Efficacy and safety of the human glucagonlike peptide- 1 analog liraglutide in combination with metformin and thiazolidinedione in patients with Type 2 diabetes (LEAD-4 Met+TZD)". Diabetes Care 32 (2009): 1224-1230.

16. A Mehta. "Liraglutide for weight management: a critical review of the evidence" (2017).

17. Brown-Frandsen K., et al. "Lower rates of cardiovascular events and mortality associated with liraglutide use in patients treated with basal insulin: A DEVOTE subanalysis (DEVOTE 10)" (2019).

18. Xu Zhao., et al. "Effect of liraglutide on blood pressure: a metaanalysis of liraglutide randomized controlled trials" (2019).

19. Plainsboro NJ. Saxenda [package insert]. Novo Nordisk: (2015).

20. WM Steinberg., et al. EPub Ahead of Print. Diabetes Care (2017).

Volume 3 Issue 10 October 2019

(C) All rights are reserved by Tarek Elshourbagy. 